\title{
Connectivity Forests for Homological Analysis of Digital Volumes*
}

\author{
Pedro Real \\ Dpto. Matematica Aplicada I, E.T.S.I. Informatica, \\ Universidad de Sevilla, \\ Avda. Reina Mercedes, s/n 41012 Sevilla (Spain) \\ real@us.es
}

\begin{abstract}
In this paper, we provide a graph-based representation of the homology (information related to the different "holes" the object has) of a binary digital volume. We analyze the digital volume AT-model representation 8] from this point of view and the cellular version of the AT-model [5] is precisely described here as three forests (connectivity forests), from which, for instance, we can straightforwardly determine representative curves of "tunnels" and "holes", classify cycles in the complex, computing higher (co)homology operations,... Depending of the order in which we gradually construct these trees, tools so important in Computer Vision and Digital Image Processing as Reeb graphs and topological skeletons appear as results of pruning these graphs.
\end{abstract}

Keywords: digital volume, cell complex, chain homotopy, graph, tree, forest, homology, cohomology.

\section{Introduction}

Finding a concise, yet geometrically and topologically faithful digital representation of a digital volume is at the core of several research themes in graphics. We propose a new strategy for the design of succinct and efficient volume approximations. In [5], given a binary 26-adjacency voxel-based digital volume $V$, the homological information (that related to $n$-dimensional holes: connected components, "tunnels" and cavities) is extracted from a linear map (called homology gradient vector field) acting on a polyhedral cell complex $P(V)$ homologically equivalent to $V$. Based on that description, we define here a three-level graph data structure for representing digital volumes. This connectivity encoding technique attempt to reduce the redundancy inherent in many popular representations of polyhedral or triangular meshes in $3 \mathrm{D}$.

In this paper, we first develop an algebraic homological study for dealing with special chain homotopies appearing in the technique developed in [5] for determining homology gradient vector fields for a binary voxel-based digital volume.

* This work has been partially supported by "Computational Topology and Applied Mathematics" PAICYT research project FQM-296, "Andalusian research project PO6-TIC-02268 and Spanish MEC project MTM2006-03722. 
From this study, we derive a graph-based representation of homology gvfs and, finally, the problem of encoding the connectivity in $3 \mathrm{D}$ is solved here using a hierarchical "cover" forest describing the homology of a cell continuous analogous (cell AT-model) of the volume.

\section{Chain Homotopies and Gradient Vector Fields}

We work here with 3-dimensional cell complexes. The cells are typically polyhedra, polygons, line segments and points, in decreasing order of degrees. For convenience, we call a cell of degree $p$ a $p$-cell. Since the objects considered in this paper are embedded in $\mathbf{R}^{3}$ then the homology groups vanish for dimensions greater than 3 and they are torsion-free for dimensions 0,1 and 2. Throughout the paper, we consider that the ground ring is the finite field $\mathbf{F}_{2}=\{0,1\}$. Let $K$ be a three-dimensional cell complex. A $q-$ chain $a$ is a formal sum of simplices of $K^{(q)}$. We denote $\sigma \in a$ if $\sigma \in K^{(q)}$ is a summand of $a$. The $q$-chains form a group with respect to the component-wise addition; this group is the qth chain group of $K$, denoted by $C_{q}(K)$. There is a chain group for every integer $q \geq 0$, but for a complex in $\mathbf{R}^{3}$, only the ones for $0 \leq q \leq 3$ may be non-trivial. The boundary map $\partial_{q}: C_{q}(K) \rightarrow C_{q-1}(K)$ applied to a $q$-cell $\sigma$ gives us the collection of all its $(q-1)$-faces which is a $(q-1)$-chain. By linearity, the boundary operator $\partial_{q}$ can be extended to $q$-chains. For the rest of the paper, we adopt the notation $(K, \partial)$ for representing a $3 \mathrm{D}$ cell complex. In the concrete case of a simplicial complex, the boundary of a $q$-simplex defined in terms of vertices $\sigma=\left\langle v_{0}, \ldots, v_{q}\right\rangle$ is defined by: $\partial_{q}(\sigma)=\sum\left\langle v_{0}, \ldots, \hat{v}_{i}, \ldots, v_{q}\right\rangle$, where the hat means that vertex $v_{i}$ is omitted. In our case, taking into account that the 3 -cells of our cell complexes can automatically be subdivided into tetrahedra, its boundary map can directly be derived from that of the component tetrahedra. It is clear that $\partial_{q-1} \partial_{q}=0$. A chain $a \in C_{q}$ is called a $q-$ cycle if $\partial_{-} q(a)=0$. If $a=\partial_{q+1}\left(a^{\prime}\right)$ for some $a^{\prime} \in C_{q+1}$ then $a$ is called a $q$-boundary. Define the qth homology group to be the quotient group of $q$-cycles and $q$-boundaries, denoted by $H_{q}(\mathcal{C})$. Let $\mathcal{C}=\left\{C_{q}, \partial_{q}\right\}$ and $\mathcal{C}^{\prime}=\left\{C_{q}^{\prime}, \partial_{q}^{\prime}\right\}$ be two chain complexes. A chain map $f: \mathcal{C} \rightarrow \mathcal{C}^{\prime}$ is a family of homomorphisms $\left\{f_{q}: C_{q} \rightarrow C_{q}^{\prime}\right\}_{q \geq 0}$ such that $\partial_{q}^{\prime} f_{q}=f_{q-1} \partial_{q}$.

The following definitions are necessary in order to classify gradient vector fields.

Definition 1. [3] Let $(K, \partial)$ be a finite cell complex. A linear map of chains $\phi: C_{*}(K) \rightarrow C_{*+1}(K)$ is a combinatorial gradient vector field (or, shortly, combinatorial gvf) over $K$ if the following conditions hold:

1. For any cell $a \in K_{q}, \phi(a)$ is a $q+1$-cell $b$.

2. $\phi^{2}=0$

If we remove the first condition, then $\phi$ will be called an algebraic gradient vector field. If $\phi$ is a combinatorial gvf which is only non-null for a unique cell $a \in K_{q}$ and satisfying the extra-condition $\phi \partial \phi=\phi$, then it is called a (combinatorial) integral operator [6]. An algebraic gvf satisfying the condition $\phi \partial \phi=\phi$ is called 
an algebraic integral operator. An algebraic gvf satisfying the conditions $\phi \partial \phi=\phi$ and $\partial \phi \partial=\partial$ will be called a homology gvf [5]. A gvf is called strongly-nilpotent if it satisfies the following property: given any $u \in K_{q}$, and being $\phi(u)=\sum_{i=1}^{r} v_{i}$, then $\phi\left(v_{i}\right)=0, \forall i$. We say that a linear map $f: C_{*}(K) \rightarrow C_{*}(K)$ is strongly null over an algebraic gradient vector field $\phi$ if given any $u \in K_{q}$, and being $\phi(u)=\sum_{i=1}^{r} v_{i}$, then $f\left(v_{i}\right)=0, \forall i$.

Using homological algebra arguments, it is possible to deduce that a homology gvf $\phi$ over $K$ determines a strong algebraic relationship connecting $C(K)$ and its homology vector space $H(K)$. Let us define a chain contraction $(f, g, \phi)$ : $(C, \partial)=>\left(C^{\prime}, \partial^{\prime}\right)$ between two chain complexes as a triple of linear maps such that $f: C_{*} \rightarrow C_{*}^{\prime}, g: C_{*}^{\prime} \rightarrow C_{*}$ and $\phi: C_{*} \rightarrow C_{*+1}$ and they satisfy the following conditions: (a) $i d_{C}-g f=\partial \phi+\phi \partial ;(b) f g=i d_{C^{\prime}}$; (c) $f \phi=0$; (d) $\phi g=0$; (e) $\phi \phi=0$.

Given a chain contraction $(f, g, \phi)$, it is an elementary homological algebra result that $\operatorname{Ker} \phi=\operatorname{Im} g+\operatorname{Im} \phi$ and $\operatorname{Im} \phi$ is acyclic (i.e, it has null homology).

Proposition 1. Let $(K, \partial)$ be a finite cell complex. A homology gvf $\phi: C_{*}(K) \rightarrow$ $C_{*+1}(K)$ over $K$ give raise to a chain contraction $(f, g, \phi)=(\pi$, incl, $\phi)$ from $C(K)$ onto a chain subcomplex of it isomorphic to the homology of $K$. Reciprocally, given a chain contraction $(f, g, \phi)$ from $C(K)$ to its homology $H(K)$, then $\phi$ is a homology gvf.

Let incl $: \operatorname{Im} \pi \rightarrow C(K)$ be the inclusion map. Let $\pi=i d_{\mathcal{C}(\mathcal{K})}-\partial \phi-\phi \partial$. This chain map describe for each cell a representative cycle of the homology class associated to this cell and satisfies that $\pi^{2}=\pi$. If $\operatorname{Im} \pi=\{x \in C(K)$, such that $x=$ $\phi(y)$ for some $y\}$ and $\operatorname{Ker} \pi=\{x \in C(K)$ such that $\phi(x)=0\}$, then $\mathcal{C}(\mathcal{K})=$ $\operatorname{Im} \pi \oplus \operatorname{Ker} \pi)$. Let $f: C(K) \rightarrow \operatorname{Im}(\pi)$ be the corestriction of $\pi$ to $\operatorname{Im}(\pi)$ (that is, $\pi: C(K) \rightarrow \operatorname{Im}(\pi))$ and $g: \operatorname{Im}(\pi) \rightarrow C(K)$ be the inclusion. Let $\tilde{\partial}$ be the boundary operator of $\operatorname{Im}(\pi)$. We now prove that $\tilde{\partial}=0$. Taking into account that $i d_{C(K)}+g f=\phi \partial+\partial \phi, \partial \partial=0$ and $\partial \phi \partial=\partial$, we then obtain $\partial-\partial g f=\partial$. Therefore, $\partial g f=g \tilde{\partial} f=0$. Since $f$ is onto and $g$ is one-to-one, we deduce that $\tilde{\partial}=0$. That means that the so-called Morse complex associated to $\phi, M_{\phi, \partial}=\operatorname{Im} \pi$ is a graded vector space with null boundary operator isomorphic to the homology $H(K)$.

The following proposition can be seen as an elementary boundary-perturbation result.

Proposition 2. Let $(K, \partial)$ be a finite cell complex and $\phi: C_{*}(X) \rightarrow C_{*+1}(X)$ be a homology gvf over $K$. If we consider a new boundary map $\partial+\delta$ for $C_{*}(X)$ (that is, $\delta: C_{*}(K) \rightarrow C_{*-1}(K)$ is a linear map satisfying $\left.(\partial+\delta)^{2}=0\right)$ such that $\phi \delta \phi=0$, then a new chain contraction $\left(f_{\delta}, g, \phi\right)=(\pi+\phi \delta+\delta \phi$, incl, $\phi)$ from $C_{*}(K)$ onto the Morse complex $M_{\phi, \partial+\delta}=\operatorname{Im}(\pi+\phi \delta+\delta \phi)$ (having in general, a non-null boundary map $\delta+\partial \phi \delta+\delta \phi \partial+\delta \phi \delta)$ can be established.

It is clear that

Proposition 3. In the conditions of Proposition 2 and if $\delta \phi=0$, then the boundary map of $\mathcal{M}_{\phi, \partial+\delta}$ is $\delta+\partial \phi \delta$. 
Naming $\pi^{\prime}=\pi+\phi \delta+\delta \phi$ and being in general $\phi \delta \phi \neq 0$, then we have that $i d_{C(K)}+g f=\phi d+d \phi$ and, for an element $x=\pi^{\prime}(y)$, such that $\pi^{\prime}(y) \neq\left(\pi^{\prime}\right)^{2}(y)$ then

$$
x+\pi^{\prime}(x)=(\partial+\delta) \phi \pi^{\prime}(y)+\phi \pi^{\prime}(\partial+\delta)(y) .
$$

In fact, an algebraic integral operator (non necessarily a homology gvf) $\phi$ over $K$ always determines a chain contraction from $C(K)$ to $\operatorname{Im} \pi$ (Morse complex having in general a non-null boundary). If $\phi$ is an algebraic gvf which does not satisfy in general the condition $\phi d \phi=\phi$, then we have $i d_{C(K)}+g f=\phi d+d \phi$ and, for an element $x=\pi(y)$, such that $\pi(y) \neq \pi^{2}(y)$ then

$$
x+f g(x)=\partial \phi \pi(y)+\phi \pi \partial(y) .
$$

Now, we can easily prove the following gvf-perturbation result:

Proposition 4. Let $(K, \partial)$ be a finite cell complex and $\phi: C_{*}(K) \rightarrow C_{*+1}(K)$ be an algebraic gvf over $K$. If we consider a new algebraic gvf $\phi+\psi$ (that is, $\psi: C_{*}(K) \rightarrow C_{*+1}(K)$ is a linear satisfying that $\left.(\phi+\psi)^{2}=0\right)$ such that $(\phi+\psi)+(\phi+\psi) \partial(\phi+\psi)=0$, then a new chain contraction $\left(f_{\psi}, g, \phi+\psi\right)=$ $(\pi+\psi \partial+\partial \psi$, incl, $\phi)$ from $C_{*}(K)$ onto the Morse complex $\mathcal{M}_{\phi+\psi, \partial}=\operatorname{Im}(\pi+$ $\psi \partial+\partial \psi)$ (having in general, a non-null boundary map $\partial+\partial(\phi+\psi) \partial)$ can be established.

It is time to analyze an incremental technique for getting gvfs, using the previous elementary perturbation steps. In this way, the incremental technique gives us a combinatorial gvf. Given a cell complex $(K, \partial)$, the ordered set of cells $\mathcal{K}=\left\langle c_{1}, \ldots, c_{m}\right\rangle$ is a filter if all the faces of the cell $c_{j}$ belong to the subset $\left\langle c_{1}, \ldots, c_{j-1}\right\rangle$. It is possible to "filter" $K$ by first considering all the 0 -cells in a certain order, then an order on all the 1-cells, and so on.

Algorithm 1. Let $(K, \partial)$ be a finite cell complex with filter $\mathcal{K}_{m}=\left\langle c_{0}, \ldots, c_{m}\right\rangle$. We represent the cell complex $K$ up to filter level $i$ by $\mathcal{K}_{i}=\left\langle c_{0}, \ldots, c_{i}\right\rangle$, with boundary map $\partial_{i}$. Let $\mathcal{M}_{i}$ be a chain complex associated to $\mathcal{K}_{i}$.

$\mathcal{M}_{0}:=\left\{c_{0}\right\}, \phi_{0}\left(c_{0}\right):=0$.

For $i=1$ to $m$ do

$\mathcal{M}_{i}:=\mathcal{M}_{i-1} \cup\left\{c_{i}\right\}, \phi_{i}\left(c_{i}\right):=0$,

If $\left(\partial_{i}+\partial_{i-1} \phi_{i-1} \partial_{i}\right)\left(c_{i}\right)=0$, then

For $j=0$ to $i-1$ do, $\phi_{i}\left(c_{j}\right):=\phi_{i-1}\left(c_{j}\right)$.

If $\left(\partial_{i}+\partial_{i-1} \phi_{i-1} \partial_{i}\right)\left(c_{i}\right)$ is

a sum of a kind $\sum_{j=1}^{r} u_{j} \neq 0 \quad\left(u_{i} \in \mathcal{M}_{i-1}\right)$, then:

Let us choose one $c_{r}^{i}$ from all the summands $u_{k}=f_{i}\left(c_{k}^{i}\right)$

$=\left(1+\phi_{i-1} \partial+\partial \phi_{i-1}\right)\left(c_{k}^{i}\right)$ such that $\phi_{i-1}\left(c_{r}^{i}\right)=0$

and $c_{r}^{i} \notin \operatorname{Im} \phi_{i-1}$ and define $\phi_{i}\left(c_{r}^{i}\right):=c_{i}$

and $\phi_{i}:=\phi_{i-1}$ for the rest of elements of $\mathcal{M}_{i-1}$.

OUTPUT: a combinatorial gradient vector field $\phi_{m}$ for $K$. 
The previous algorithm can be considered as an application of discrete Morse techniques 3 . The following figure shows in a pictorial way the result of the algorithm of a concrete cell complex. The gvf $\phi_{m}$ does not satisfy in general that $\phi_{m} \partial_{m} \phi_{m}=\phi_{m}$ and it is necessary to "correct" this fact in order to get a homology gvf.

Proposition 5. Let $(K, \partial)$ a finite cell complex and $\phi: C_{*}(K) \rightarrow C_{*+1}(K)$ a gvf such that $\phi \partial \phi \neq \phi$. Then, starting from $\phi$ it is possible to construct a chain contraction connecting $C(K)$ and $\operatorname{Im}^{n}$ (Morse cellular complex associated to the gvf $\phi)$, where $\pi=1+\partial \phi+\phi \partial$.

Proof. If $\phi \partial \phi \neq \phi$ for a gvf $\phi$ over $(K, \partial)$, the translation of Forman's work [3] to the chain homotopy language is as follows:

$$
\begin{gathered}
1+\pi=\phi \partial+\partial \phi \\
\pi+\pi^{2}=\pi \phi \partial+\partial \pi \phi \\
\cdots \cdots \\
\pi^{n-1}+\pi^{n}=\pi^{n-1} \phi \partial+\partial \pi^{n-1} \phi \\
\pi^{n}+\pi^{n+1}=\pi^{n} \phi \partial+\partial \pi^{n} \phi
\end{gathered}
$$

Let us suppose that $\pi^{n}=\pi^{n+1}$, that is, $\pi^{n}(1+\pi)=0$. This property, called stabilization by Forman, can be satisfied, for example if $(1+\partial \phi)^{n}=0$. Then, we get that

$$
1+\pi^{n}=\left(\sum \pi^{k}\right) \phi \partial+\partial\left(\sum \pi^{k}\right) \phi
$$

and it is not difficult to prove that $\left(\pi^{n}, i n c l,\left(\sum_{k=0}^{n} \pi^{k}\right) \phi\right)$ is a chain contraction connecting $C(K)$ and $\operatorname{Im} \pi^{n}$ (Morse cellular complex associated to the gvf $\phi$ ).

\section{Graph Representation of a Homology Gradient Vector Field}

Using Discrete Morse Theory and its pictorial language, combinatorial gvfs can be described in terms of directed graphs on the cell complex. For example, let us take an integral operator $\phi$ such that $\phi(a)=c, a \in K_{0}$ and being $a$ and $b$ the vertices of the 1-cell $c$. It is clear that $\phi$ can be represented by a directed tree consisting in the edge $c$ together with its vertices, such that the arrow on $c$ goes out from vertex $a$. Of course, the previous properties of a homology gvf $\phi_{i}: C_{i}(K) \rightarrow C_{i+1}(K)(i=0,1,2)$ help us to suitably express all the $\phi_{i}$ in terms of graphs.

Proposition 6. If $\phi: C(K) \rightarrow C(K)$ is a homology gvf for a cell complex $(K, \partial)$ and we denote by $H^{\partial}(K)$ and $H^{\phi}(K)$ the homology groups of $K$ taking respectively $\partial$ and $\phi$ as boundary maps on $K$ (both satisfy the 2-nilpotency condition). Then, $H^{\partial}(K)$ and $H^{\phi}(K)$ are isomorph. The maps $h: H^{\partial}(K) \rightarrow H^{\phi}(K)$ defined by $h\left([c]^{\partial}\right)=[c+\partial \phi(c)]^{\phi}$ and $k: H^{\phi}(K) \rightarrow H^{\partial}(K)$ defined by $h\left([c]^{\phi}\right)=$ $[c+\phi \partial(c)]^{\phi}$ specify this isomorphism. 
The following homology computation algorithm is given in [5]. It describes each incremental step as a composition of chain contractions and provides a easy way to prove that the final chain homotopy $\phi_{m}$ is strongly nilpotent.

Given a cell complex $(K, \partial)$, the ordered set of cells $\mathcal{K}=\left\langle c_{1}, \ldots, c_{m}\right\rangle$ is a filter if $c_{i}$ is a face of $c_{j}$ for $i<j$. It is possible to "filter" $K$ by first considering all the 0 -cells in a certain order, then an order on all the 1-cells, and so on.

Algorithm 2. Let $(K, \partial)$ be a finite cell complex with filter $\mathcal{K}_{m}=\left\langle c_{0}, \ldots, c_{m}\right\rangle$. We represent the cell complex $K$ up to filter level $i$ by $\mathcal{K}_{i}=\left\langle c_{0}, \ldots, c_{i}\right\rangle$, with boundary map $\partial_{i}$. Let $\mathcal{H}_{i}$ the homology chain complex (with zero boundary map) associated to $\mathcal{K}_{i}$.

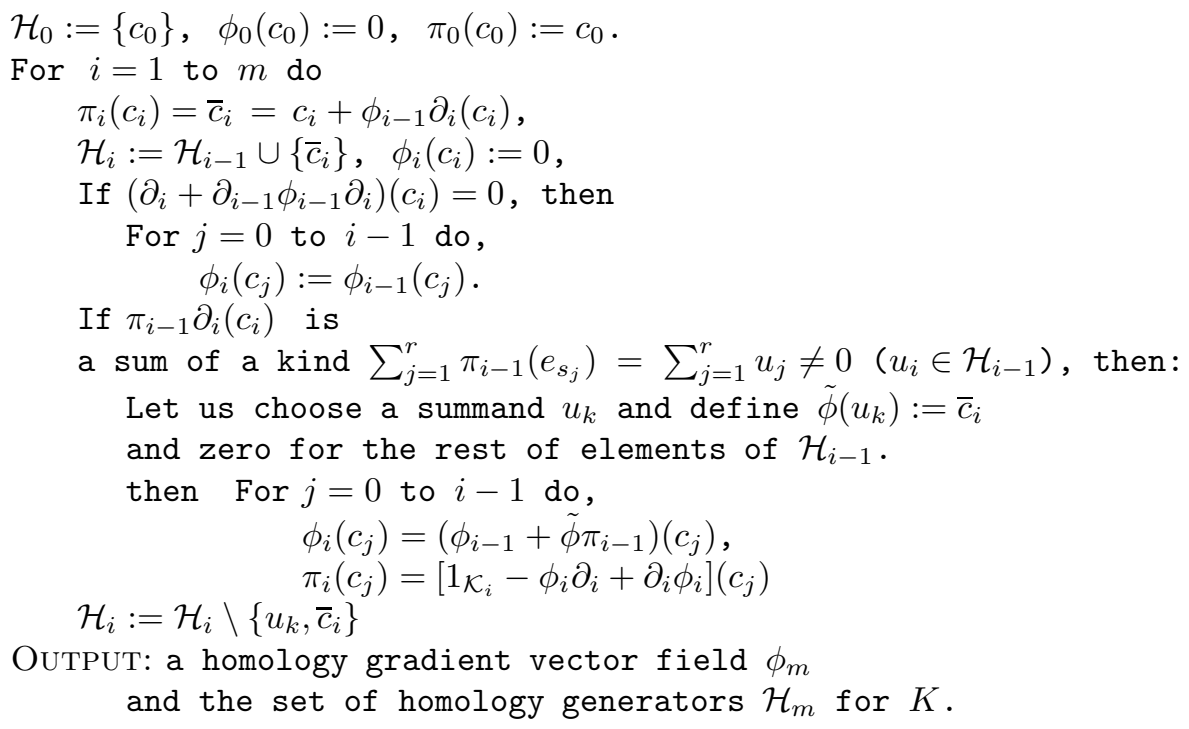

The following result that can be easily proved using induction on $i$ is the key for determining the graph nature of a homology gradient vector field
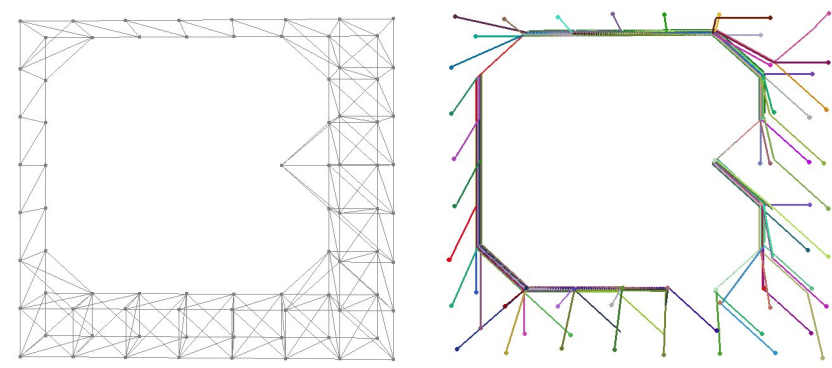

Fig. 1. Zoom of the polyhedral cell complex associated to a digital volume and its associated vertex tree 
Proposition 7. In the previous algorithm, the homology gradient vector field $\phi_{m}$ is strongly nilpotent and the map $\pi_{m}=1+\partial \phi_{m}+\phi_{m} \partial$ is strongly null over $\phi_{m}$.

Proposition 8. Let $(K, \partial)$ a finite cell complex and $\phi$ a strongly nilpotent homology gradient vector field on $K$ with map $\pi=1+\partial \phi+\phi \partial$ being strongly null over $\phi$. Then, the following properties hold:

1. $\phi(u)$ can be represented as a path from $u$ to a fixed $u_{0}=(1+\phi d+d \phi)(u)$.

2. If $\phi(u)=u v+\tilde{\phi}(u)$, then $\phi(v)=\tilde{\phi}(u)$.

3. The graph of consisting of all the paths $\phi(u), u \in K_{0}$ is a forest (as many trees as connected components the object has).

4. If $\partial \phi\left(c-c^{\prime}\right)=0$, then $\phi(c)=\phi\left(c^{\prime}\right), \forall c, c^{\prime} \in K_{i}, \quad i=0,1,2$.

In fact, these properties and the fact that $\operatorname{Im} g$ has null homology guarantee that $\operatorname{Im} \phi$ in each level (levels 0 determined by the set $\phi\left(C_{0}(K)\right)$, level 1 by $\phi\left(C_{1}(K)\right)$ and level 2 by $\left.\phi\left(C_{2}(K)\right)\right)$ can be represented as a kind of "cover" forest $\left(F(\phi)_{0}, F(\phi)_{1}, F(\phi)_{2}\right)$ for the cell complex $K$. From level 0 to 1 , the 1cells appearing as summands in $\phi\left(C_{0}(K)\right)$ are the edges of the graph $F(\phi)_{0}$ and all the 0-cells are the vertices. In fact, $F(\phi)_{0}$ is a spanning forest of the graph defined by the vertices and edges of the cell complex $K$ and it is called vertex forest. From level 1 to 2 , the rest of 1-cells of $K$ not involved in $F(\phi)_{0}$ are the vertices of the graph $F(\phi)_{1}$ (in fact, each edge is represented by its barycenter) and the 2-cells which are summands in $\phi\left(C_{1}(K)\right)$ generates the edges of $F(\phi)_{1}$ (in fact, they join the "vertices" of the graph $\left.F(\phi)_{1}\right)$. The critical 1-cells (that is, the 1-cells $u$ of $K$ such that $\phi(u)=0$ )are the vertices of $F(\phi)_{1}$ which are isolated. If there is a 2-cell $v=\phi(u)$, for some 1-cell $u$ such that the rest of 1-cells of its boundary belong to the set of edges of $F(\phi)_{0}$ or to the set of critical 1-cells, then $v$ is represented in $F(\phi)_{1}$ by an edge connecting the vertex $u$ with the barycenter of $v$.

From level 2 to 3, the rest of 2-cells of $K$ not involved in $F(\phi)_{1}$ are the vertices of the graph $F(\phi)_{2}$ (each 2-cell is represented by its barycenter) and all the 3cells which are summands in $\phi\left(C_{2}(K)\right)$ generate the edges connecting them (if two 2-cells share a 3-cell, then these "vertices" are connected by an edge). The critical 2-cells (that is the 2-cells $u$ of $K$ such that $\phi(u)=0$ )are the vertices of $F(\phi)_{2}$ which are isolated. If there is a 3-cell $v=\phi(u)$, for some 2-cell $u$ such that the rest of 2-cells of its boundary belong to the set of edges of $F(\phi)_{1}$ or to the set of critical 2-cells, then $v$ is represented in $F(\phi)_{2}$ by an edge connecting the vertex $u$ with the barycenter of $v$.

\section{Trees Encoding the Connectivity of Digital Volumes}

In [5], given a binary 26-adjacency voxel-based digital volume $V$, the homological information (that related to $n$-dimensional holes: connected components, "tunnels" and cavities) is extracted from a linear map (called homology gradient vector field) acting on a polyhedral cell complex $P(V)$ homologically equivalent 

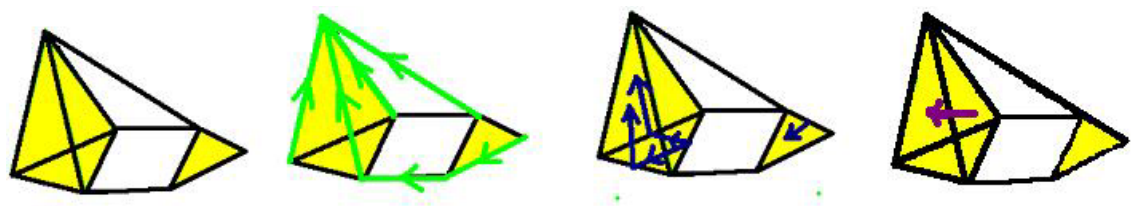

Fig. 2. A cell complex $K$ and its corresponding forests $F(\phi)_{0}, F(\phi)_{1}$ and $F(\phi)_{2}$

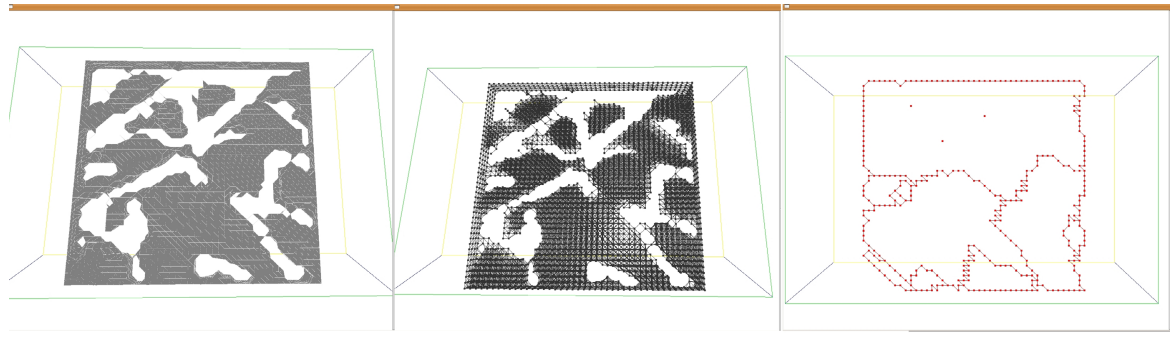

Fig. 3. Topological skeleton of a trabecular bone

to $V$. As we have seen in the previous sections, this map can be represented with no loss of information by three forests. This technique can be considered as an appropriate generalization of the spanning tree technique (arising from the graph theory) to three dimensions 13 .

For a 3D binary digital image $V$, the choice of the filter and the choice of the pair of cells in each step of the Algorithm 2 allows us to see Reeb graphs, topological skeletons or other topological tools of $V$ as results of pruning in the connectivity forests determining the homology gradient vector field.

\section{References}

1. Damiand, G., Peltier, S., Fuchs, L., Lienhardt, P.: Topological Map: An Efficient Tool to Compute Incrementally Topological Features on 3D Images. In: Reulke, R., Eckardt, U., Flach, B., Knauer, U., Polthier, K. (eds.) IWCIA 2006. LNCS, vol. 4040, pp. 1-15. Springer, Heidelberg (2006)

2. Delfinado, C.J.A., Edelsbrunner, H.: An Incremental Algorithm for Betti Numbers of Simplicial Complexes on the 3-Sphere. Comput. Aided Geom. Design 12, 771784 (1995)

3. Forman, R.: A Discrete Morse Theory for Cell Complexes. In: Yau, S.T. (ed.) Geometry, Topology \& Physics for Raoul Bott. International Press (1995)

4. Gonzalez-Diaz, R., Medrano, B., Real, P., Sanchez-Pelaez, J.: Algebraic Topological Analysis of Time-sequence of Digital Images. In: Ganzha, V.G., Mayr, E.W., Vorozhtsov, E.V. (eds.) CASC 2005. LNCS, vol. 3718, pp. 208-219. Springer, Heidelberg (2005)

5. Molina-Abril, H., Real, P.: Advanced homological information of digital volumen via cell complexes. In: da Vitora Lobo, N., et al. (eds.) SSPR\&SPR 2008. LNCS, vol. 5342, pp. 361-371. Springer, Heidelberg (2008) 
6. Gonzalez-Diaz, R., Jimenez, M.J., Medrano, B., Molina-Abril, H., Real, P.: Integral Operators for Computing Homology Generators at Any Dimension. In: RuizShulcloper, J., Kropatsch, W.G. (eds.) CIARP 2008. LNCS, vol. 5197, pp. 356-363. Springer, Heidelberg (2008)

7. Gonzalez-Diaz, R., Jiménez, M.J., Medrano, B., Real, P.: Chain Homotopies for Object Topological Representations. Discrete and Applied Mathematics 157, 490499 (2009)

8. Gonzalez-Diaz, R., Real, P.: On the Cohomology of $3 D$ Digital Images. Discrete Applied Math. 147, 245-263 (2005)

9. Hatcher, A.: Algebraic Topology. Cambridge University Press, Canbridge (2001)

10. Munkres, J.R.: Elements of Algebraic Topology. Addison-Wesley Co., Reading (1984)

11. Peltier, S., Alayrangues, S., Fuchs, L., Lachaud, J.O.: Computation of homology groups and generators. Computers \& Graphics 30(1), 62-69 (2006)

12. Peltier, S., Ion, A., Haxhimusa, Y., Kropatsch, W.G., Damiand, G.: Computing Homology Group Generators of Images Using Irregular Graph Pyramids. In: Escolano, F., Vento, M. (eds.) GbRPR 2007. LNCS, vol. 4538, pp. 283-294. Springer, Heidelberg (2007)

13. Suuriniemi, S., Tarhasaari, T., Kettunen, L.: Generalization of the spanning-tree technique. IEEE Transactions on Magnetics 38(2), 525-528 (2002)

14. Taubin, G., Rossignag, J.: Geometric compression through topological surgery. ACM Transactions on Graphics 17(2), 84-115 (1998) 